\title{
Comparison between OpenFOAM CFD \& BEM theory for variable speed - variable pitch HAWT
}

\author{
Islam ElQatary ${ }^{1, \mathrm{a}}$ and Basman Elhadidi ${ }^{2, \mathrm{~b}}$ \\ ${ }^{1}$ Research Assistant at ASU-GARDS Ain Shams University and Department of Aerospace, \\ Cairo University \\ ${ }^{2}$ Associate Professor at Department of Aerospace, Cairo University
}

\begin{abstract}
OpenFoam is used to compare computational fluid dynamics (CFD) with blade element momentum theory (BEM) for a variable speed - variable pitch HAWT (Horizontal Axis Wind Turbine). The wind turbine is first designed using the BEM to determine the blade chord, twist and operating conditions. The wind turbine blade has an outer diameter of $14 \mathrm{~m}$, uses a NACA $63-415$ profile for the entire blade and root to tip twist distribution of $15^{\mathrm{deg}}$ (Figure 3 ). The RPM varies from 20-75 for freestream velocities varying between $3-10.5 \mathrm{~m} / \mathrm{s}$ (variable speed) and a constant RPM of 78.78 for velocities ranging between $11-25 \mathrm{~m} / \mathrm{s}$ (variable pitch). OpenFOAM is used to investigate the wind turbine performance at several operating points including cut-in wind speed $(3 \mathrm{~m} / \mathrm{s})$, rated wind speed $(10.5 \mathrm{~m} / \mathrm{s})$ and in the variable pitch zone. Simulation results show that in the variable-speed operating range, both CFD and BEM compare reasonably well. This agreement can be attributed to the fact that the complex three-dimensional flow around the turbine blades can be split into two radial segments. For radii less than the mid-span, the flow is three-dimensional, whereas for radii greater than the mid-span, the flow is approximately two-dimensional. Since the majority of the power is produced from sections beyond the mid-span, the agreement between CFD and BEM is reasonable. For the variable-pitch operating range the CFD results and BEM deviate considerably. In this case the majority of the power is produced from the inner sections in which the flow is three-dimensional and can no longer be predicted by the BEM. The results show that differences in pitch angles up to $10^{\mathrm{deg}} \mathrm{can}$ result to regulate the power for high wind speeds in the variable-pitch operation zone.
\end{abstract}

\section{Introduction}

Wind power has been used for centuries for different purposes such as sailing, water pumping, and milling grains. In the early $19^{\text {th }}$ century wind turbines were also extended to provide electric energy. The use of wind power experienced a considerable reduction of its share in electricity production during the $20^{\text {th }}$ century because of the wide use of the more economical fossil fuels. Currently renewable energy (including wind energy) is expected to replace traditional power plants to provide cleaner energy at reasonable economic prices.

\footnotetext{
${ }^{a}$ e-mail: islamelqatary@gmail.com

be-mail: belhadid@eng.cu.edu.eg
}

This is an Open Access article distributed under the terms of the Creative Commons Attribution License 2.0, which permits unrestricted use, distribution, and reproduction in any medium, provided the original work is properly cited. 
A wind turbine converts the kinetic energy available in the wind by rotating blades and turning electric generators. The power extracted from a wind turbine is proportional to the cube of the wind speed, square of the radius and the power coefficient. To extract more power, a turbine must be larger and operate in a site with more available energy. The power coefficient of a turbine depends on the blade geometry, profile, chord and twist and must be computed from an aerodynamics model. Most turbine design codes rely on the BEM [17] to compute the power coefficient and design the first prototype turbine to predict the turbines performance in different flow regimes (wind speeds, yaw angles, setting angles of blades, etc.) and for different control zones (variable speed - variable pitch). While BEM offers the flexibility and speed to perform optimization studies to determine the optimum dimensions for a wind turbine, it assumes that the flow on the turbine blade is essentially two-dimensional with some corrections for tip effects and blade solidity. Alternatively more complex methods can be used to determine the wind turbine performance such as vortex methods $[1,2]$ and asymptotic acceleration potential methods [3]. Such models offer some improvements over BEM however with the increase in computational requirements, CFD is emerging as a tool that can be used to investigate new novel concepts for wind turbines, performance studies and perform detailed optimization studies.

Previously CFD was applied to estimate the performance of two-dimensional airfoil sections [8, 12] under different flow conditions. This was compelled by the need of developing new airfoil sections suitable for wind turbine blades. Recently, full three-dimensional analysis has been performed to simulate the flow over complete wind turbine blades (and farms) including tower effects, ground terrain effects, different blade tip shapes, wind gust and turbulence effects and aero-elastic blade flutter effects [9-13]. Duque et al. [7] used RANS computations on the two bladed NREL Phase VI rotors which is considered the benchmark problem in wind turbine simulation. Their results show that RANS calculations correlate well with experimental data. Bemudez et al. [5] studied both steady and unsteady aerodynamic effects for a HAWT for different variables such as air velocity (magnitude and direction) and rotor yaw. Ebert and Wood [6] showed that CFD results were very close to experimental data. They also concluded that CFD captured all the expected features of the flow around wind turbine rotor including root and tip vortices and the interaction between the tower and the blades wakes and examined the effect of blade tower interaction on both rotor thrust and torque. It must be mentioned that all these studies were made on the physical model of the wind turbine blade.

The full three-dimensional calculations are still very lengthy and expensive and can take considerable time particularly for preliminary design of wind turbines, blade shape optimization, and for real time power regulation and controller simulation. In this paper the accuracy of BEM is compared to CFD. CFD can include more physics that is neglected in the BEM and hence has a higher fidelity. Previously BEM and CFD were compared for a turbine operating in the variable speed range [15]. Here, the simulations are extended to include the variable pitch range which is currently the primary technique for power regulation in wind turbine industry. The BEM model is first used to optimize a $7 \mathrm{~m}$ wind turbine blade using NACA 63-415 airfoil sections. The performance of the wind turbine (power curve) and blade loading is then compared from the CFD results. A discussion of the results and conclusions then follow.

\section{Blade element momentum}

BEM theory assumes that the turbine blade is analyzed as a number of independent radial segments in the spanwise direction and applies conservation principles for both linear and angular momentum to compute the aerodynamic forces. The model assumes that there are no aerodynamic interactions between the blade segments, and that the aerodynamic forces of lift and drag can be obtained from known two-dimensional empirical or experimental data for a given airfoil section given the angle of attack and relative flow velocity (see Fig. 1). In BEM models the axial and rotational components of the 


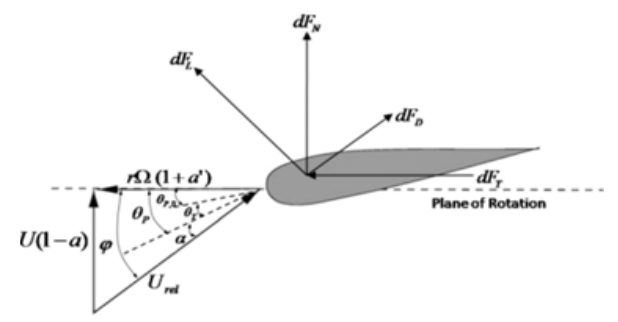

Figure 1. Blade element geometry for analysis of horizontal axis wind turbine.

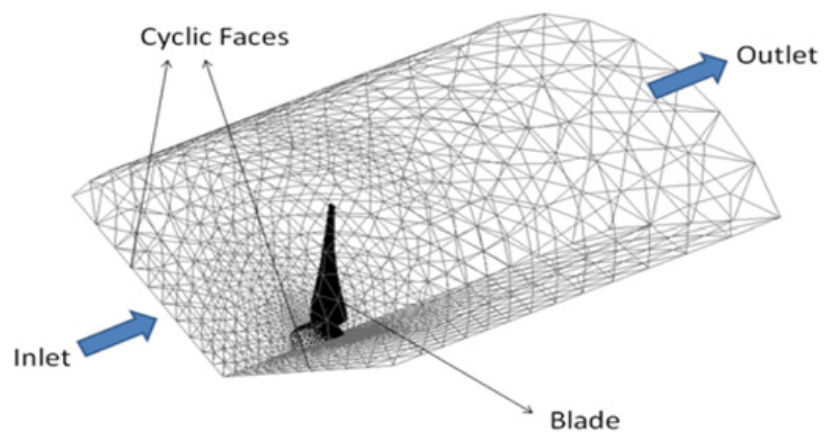

Figure 2. Mesh and boundary conditions.

air velocity are corrected by induction factors that account for the flow swirl losses, blade solidity and blockage effects. Further corrections for tip losses and solidity are incorporated in the BEM models.

The accuracy of the BEM model can be analyzed by investigating the key assumptions. First, if the axial and rotational induction factors deviate from the actual values, what impact will this have on the power? Clearly the induction factors determine the relative flow angle and hence the angle of attack. Errors in the angle of attack will result in erroneous values of the lift and drag forces and hence the integrated power. Second, can it be safely assumed that the lift and drag coefficients depend only on the two-dimensional lift and drag coefficients? This neglects the three-dimensional flow effects, flow downwash caused by the blade tips and solidity effects. The validity of such assumptions can be investigated by the use of CFD.

\section{CFD}

The modified Reynolds Averaged Navier-Stokes (RANS) equations for incompressible flow in multiple reference frames are solved in this paper (MRFSimpleFoam solver in OpenFoam). The two-equation $k-\varepsilon$ model was chosen for turbulence closure to obtain values for turbulent viscosity. This model was chosen because several comparisons of different turbulence models have been previously performed and it was concluded that the differences between them are not considerable [4] but currently there are some studies to explore the sensitivity of the turbulence modeling. The MRFSimpleFoam applies the SIMPLE algorithm together with the GAMG multi-grid solver for the pressure calculations and Gauss Seidel technique as a smoother. The residuals for the pressure were set to $10^{-3}$, velocity components to $10^{-5}$ and both $k$ and $\varepsilon$ to $10^{-7}$. The computational domain and mesh are shown in Figure 2 . The domain length is twenty times the blade radius (downstream of the blade is approximately fifteen times the blade radius). The outer radius of the annulus is five times the radius of the blade. Two meshes were generated for various pitch angles in this study. The coarser mesh has around 1.5 million 
unstructured tetrahedral elements, whereas the finer mesh has around 6 million unstructured tetrahedral elements (approximately 600,000 two-dimensional triangular elements on the wind turbine blade). The two meshes were generated (i) to compare the results of coarse and fine mesh simulations and to (ii) use the coarse mesh solution as an initial guess for the finer mesh to speed up calculations. Careful consideration is taken to limit the aspect ratio of the blade surface elements (around 3.5) to ensure that the values of the wall element $\mathrm{y}^{+}$are within the recommended ranges (3-300) for the wall-functions modeling approach. The mesh was generated using the Salome-Meca [16] software.

The inlet boundary is set to be fixed value of velocity where the major objective of this work is to validate solutions between CFD and BEM theory. The outlet boundary condition is set to be zero pressure gradients. The boundary condition of the wind turbine blade is wall boundary condition using wall functions for the turbulence model and the outer surface of the cylindrical region is set to be inletoutlet boundary condition. Cyclic boundary conditions are used on the sides of the annular domain which allows us to simulate a third of the computational domain and reduce approximately two thirds of the total computational time [14]. These boundary conditions neglect the ground effects and tower effects.

\section{Blade design using BEM}

The wind turbine used NACA 63-415 profile sections for the entire length. This section is used specifically because the pressure distribution is known experimentally for all angles of attack and can be compared to the results of CFD [15]. There are several advantages for computing the BEM before the CFD. The BEM determines the operating condition (RPM and pitch angles) of the wind turbine as a function of the inflow wind speed. This gives a preliminarily estimate for the required pitch angles and rotor RPM for the CFD simulations. If the wind turbine is allowed to rotate at any RPM the flow over the blades can suffer major separation which is not a desired state in operation.

Figure 3 presents the blade geometry, power curve, power coefficient and variable speed - variable pitch values. The airfoil sections have a constant thickness to chord ratio in this design. The cut in wind speed is $3 \mathrm{~m} / \mathrm{s}$, the rated wind speed is $10.5 \mathrm{~m} / \mathrm{s}$ and cut off wind speed is $25 \mathrm{~m} / \mathrm{s}$. It is important to note that in this design the pitch to feather was used to control the turbine beyond the rated wind speed. This translates to lower loading on the wind turbine blades and lower angles of attack. The CFD will be used to verify the operating points and performance of the wind turbine.

\section{Results}

The CFD and BEM results are compared in this section. First the power curve and pitch angles are considered. From these results it is possible to conclude the short comings of the BEM model. The deviations between the CFD and BEM are then investigated by examining the pressure distribution over the blade sections and the integral axial and tangential forces. All the results are for the fine mesh simulations. Figure 4 compares the total output power for computations on the coarse and fine mesh for different inflow wind velocities. The differences in power range from 2.2-7.7\% (using the finer mesh as the correct value). Further mesh refinement was not possible on our computational platform.

\subsection{Comparison of BEM to CFD}

Figure 5 compares the power and thrust curve obtained by the BEM with the CFD. Two sets of CFD results are plotted in each figure. The red symbols represent the simulations based on input from the BEM model (pitch angle and inlet wind speed are determined from the BEM). The blue symbols represent the corrected CFD in which the inlet wind speed was modified to regulate the power beyond the rated wind speed. From the power curve it is seen that the CFD and BEM models compare relatively 


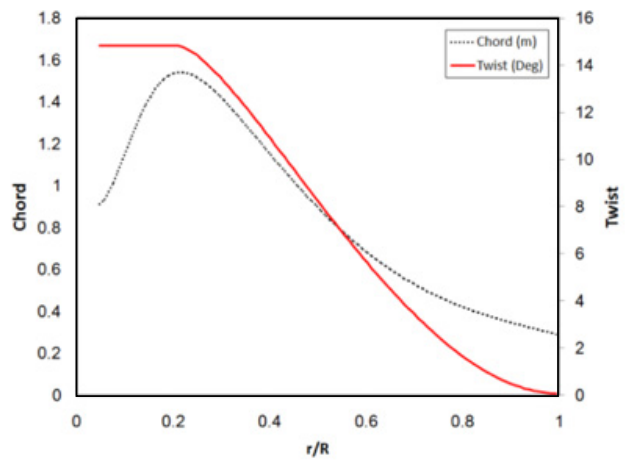

(a)

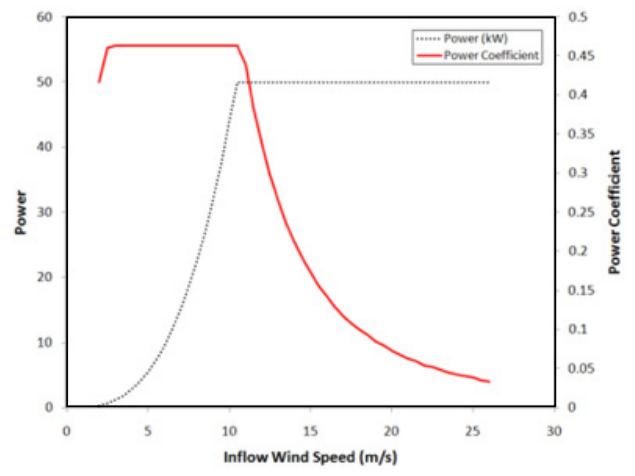

(b)

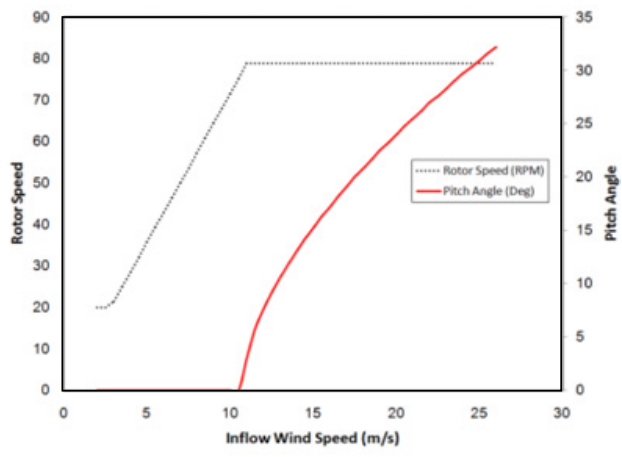

(c)

Figure 3. BEM wind turbine design. (a) Blade geometry, (b) power and coefficient of power and (c) rotor RPM and pitch angles at the root.

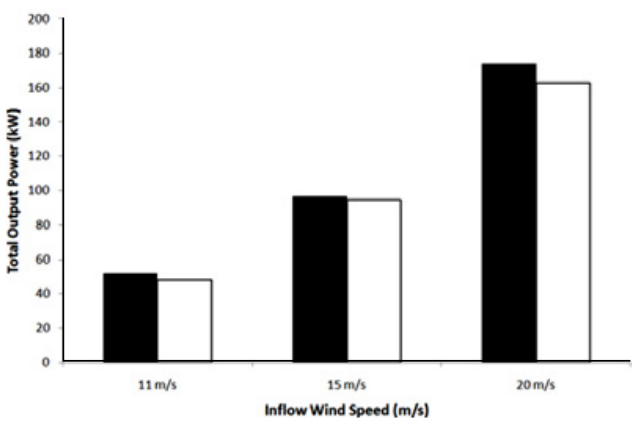

Figure 4. Comparison of coarse (left) and fine (right) mesh.

well in the variable speed zone. The agreement can be attributed to the fact that the flow over the rotor blades can be modeled as two-dimensional flow in the outer sections producing most of the power, whereas the inner complex three-dimensional flow produces less power and has a smaller effect on the blade performance [15]. In the variable pitch zone, the BEM and CFD deviate greatly. To reduce the turbine power the pitch angles were increased considerably (up to $10^{\mathrm{deg}}$ ) as seen from Figure 6 . The increase in pitch angles, results in a reduction of the relative angles of attack on the blade reducing 


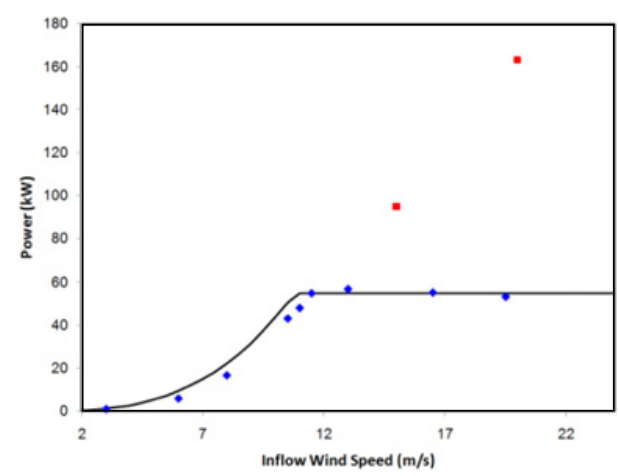

(a)

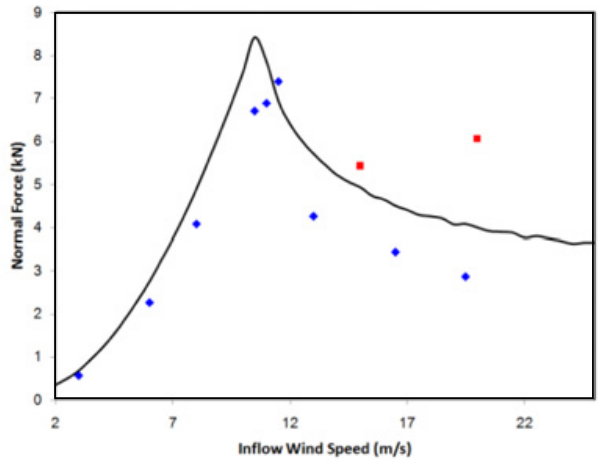

(b)

Figure 5. Power curve and thrust curve for BEM and CFD. Solid line represents BEM, blue symbols represent corrected CFD results, red symbols represent initial CFD guess.

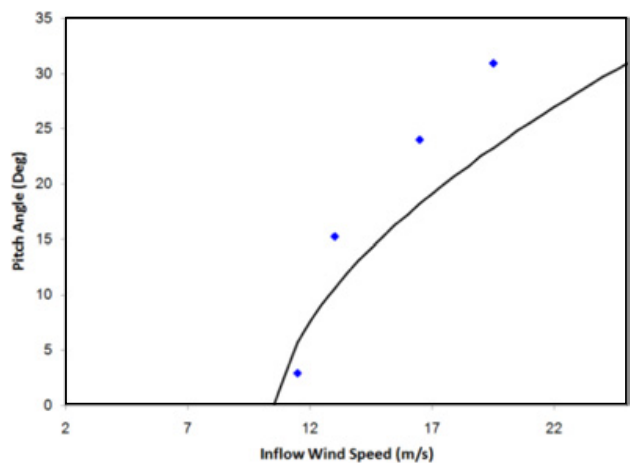

Figure 6. Pitch angle needed for regulating turbine power beyond rated wind speed. Solid line represents BEM and symbols represent corrected CFD values.

the lift and drag forces and integral thrust and power. The thrust also benefits from the increased pitch angles since the forces reduce. The trend of the thrust force is similar to that of the BEM however values are lower. In reality ground effects, tower effects and nacelle geometry can have large influences on the thrust, and full turbine geometry must be modeled to account for all of those effects.

\subsection{Assessment of CFD results}

Figure 7 shows the pressure distribution over the suction surface for the turbine blade at zero pitch for an inflow wind velocities of $8 \mathrm{~m} / \mathrm{s}$. From the figure it can be inferred that the angles of attack distribution is positive along the blade span from the higher suction pressure, and that the blade is more highly loaded towards the tip (more suction and sectional loading as will be shown in Fig. 12). Since the flow is effectively two-dimensional in the outer section, as shown in [15], the results from the CFD compare favorably to the BEM.

Figure 8 shows the pressure distribution over the suction surface for the turbine blade pitched at $15.2^{\mathrm{deg}}$ for two inflow wind velocities: (a) $13 \mathrm{~m} / \mathrm{s}$ and (b) $15 \mathrm{~m} / \mathrm{s}$. From the figure it is seen that for both inflow velocities the angles of attack are positive along the blade span. This can also be concluded from Figure 9 which compares the normal and tangential blade loading on the blade for both wind speeds 


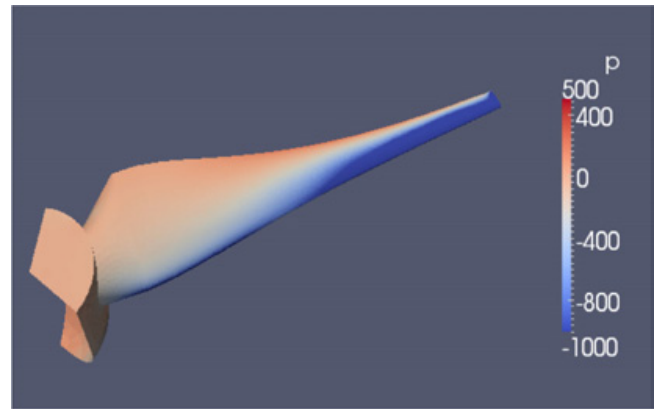

Figure 7. Pressure distribution on suction side obtained from CFD for inflow wind speed $8 \mathrm{~m} / \mathrm{s}$.

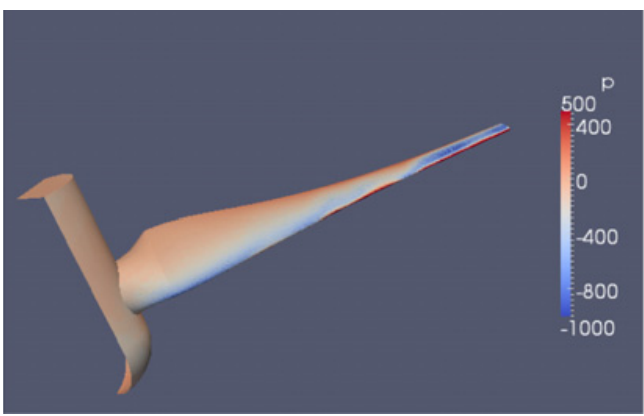

(a)

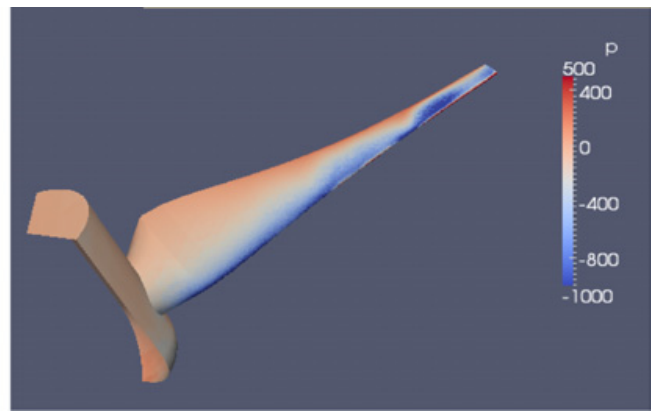

(b)

Figure 8. Comparison of pressure distribution on suction side obtained from CFD for turbine pitched at $15.2^{\mathrm{deg}}$. (a) represents corrected speed $(13 \mathrm{~m} / \mathrm{s})$ and (b) represents wind speed over predicted by BEM $(15 \mathrm{~m} / \mathrm{s})$.

with the BEM. The reduction of the velocity results in a reduction in the loads since the angles of attack reduce and available power in the wind reduces.

Figure 10 shows the pressure distribution over the suction surface for the turbine blade pitched at $24^{\mathrm{deg}}$ for two inflow wind velocities: (a) $16 \mathrm{~m} / \mathrm{s}$ and (b) $20 \mathrm{~m} / \mathrm{s}$. From the figure it is seen that for the inflow velocity predicted by the BEM model $(20 \mathrm{~m} / \mathrm{s})$ the angle of attack over the entire blade section is positive. This results in the high loading and increased power as seen in Figure 5. It is only possible to reduce the power by reducing the inflow air velocity which for the same RPM and twist angle reduces the flow angle of attack along the blade. This is seen from the pressure distribution for the case of $16 \mathrm{~m} / \mathrm{s}$, which clearly shows negative flow angles of attack beyond the mid-span. This can also be concluded from Figure 11 which compares the normal and tangential blade loading on the blade for both wind speeds with the BEM. For the lower wind speed with higher pitch angles the tip sections of the blade produce lower values of tangential force and negative power.

Figure 12 compares the normalized torque distribution (normalized by dynamic pressure and rotor area) for two CFD cases representing the variable-speed (wind speed is $8 \mathrm{~m} / \mathrm{s}$ ) and variable-pitch (wind speed is $16 \mathrm{~m} / \mathrm{s}$ ) operating zones. The power can be considered as the area underneath the curve multiplied by the rotor speed. From the figure it is clear that for the variable-speed zone the power is mostly extracted from the outer blade sections. Previously [15] it was shown that the outer blade sections can be considered as two-dimensional sections, and hence the comparison of the CFD with BEM is possible. From the figure it is also clear that for the variable-pitch zone the power is mostly extracted from the inner blade sections where the flow is three-dimensional and hence the failure of the BEM model. 


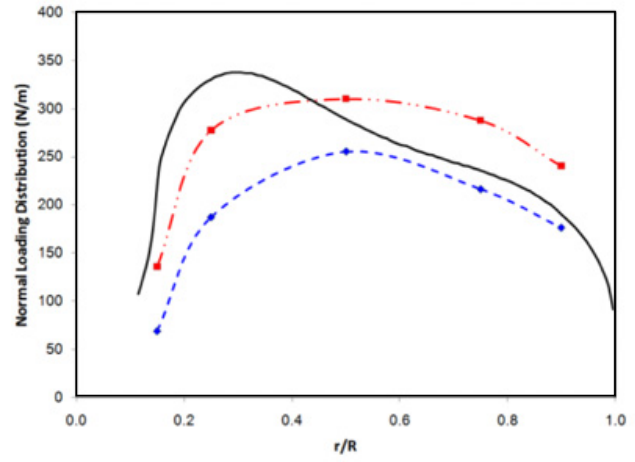

(a)

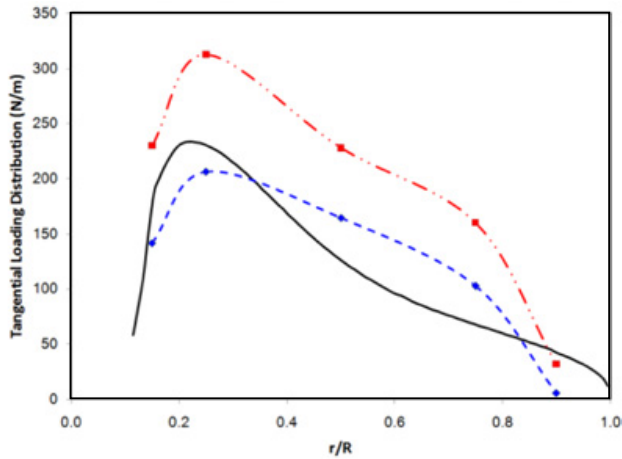

(b)

Figure 9. Comparison of normal loading (a) and tangential loading (b) between BEM (solid line) and CFD for turbine pitched at $15.2^{\mathrm{deg}}$. Dashed blue line represent corrected speed $(13 \mathrm{~m} / \mathrm{s})$ and dash-dot red lines represent wind speed over predicted by BEM $(15 \mathrm{~m} / \mathrm{s})$.

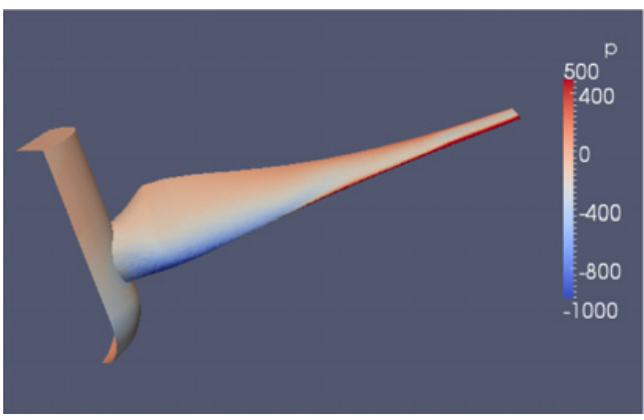

(a)

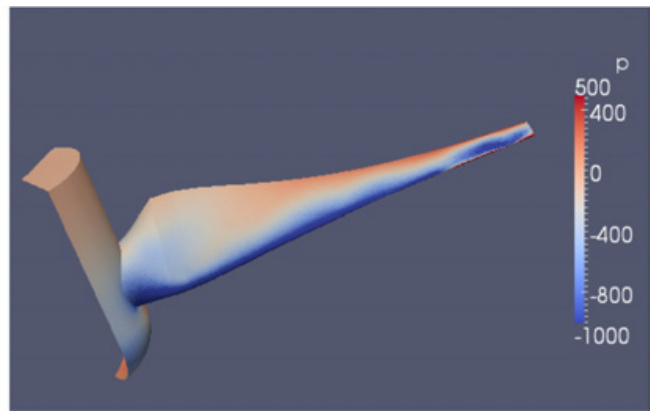

(b)

Figure 10. Comparison of pressure distribution on suction side obtained from CFD for turbine pitched at $24^{\mathrm{deg}}$. (a) represents corrected speed $(16 \mathrm{~m} / \mathrm{s})$ and (b) represents wind speed over predicted by BEM $(20 \mathrm{~m} / \mathrm{s})$.

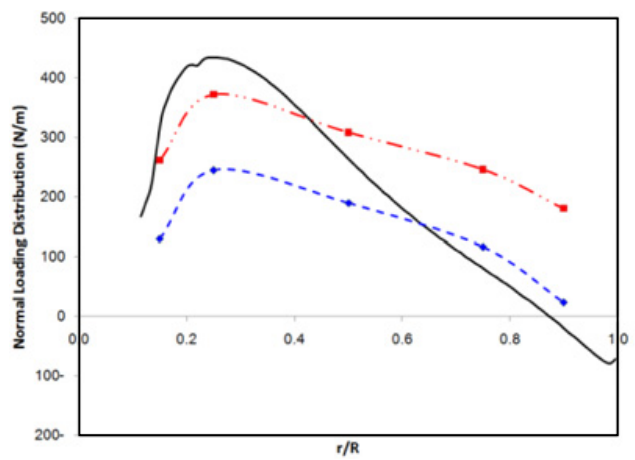

(a)

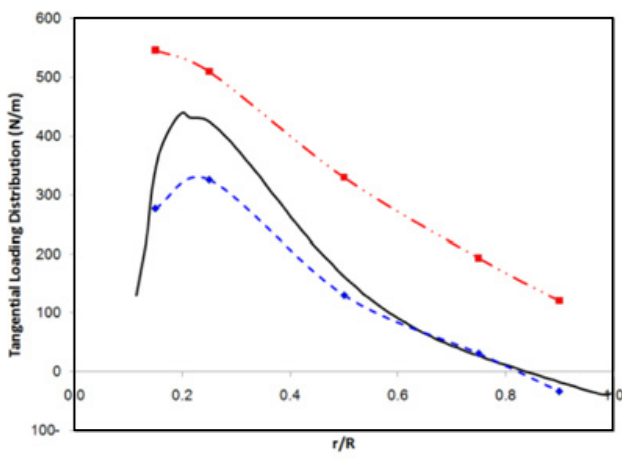

(b)

Figure 11. Comparison of normal loading (a) and tangential loading (b) between BEM (solid line) and CFD for turbine pitched at $24^{\mathrm{deg}}$. Dashed blue line represent corrected speed $(16 \mathrm{~m} / \mathrm{s})$ and dash-dot red lines represent wind speed over predicted by BEM $(20 \mathrm{~m} / \mathrm{s})$. 


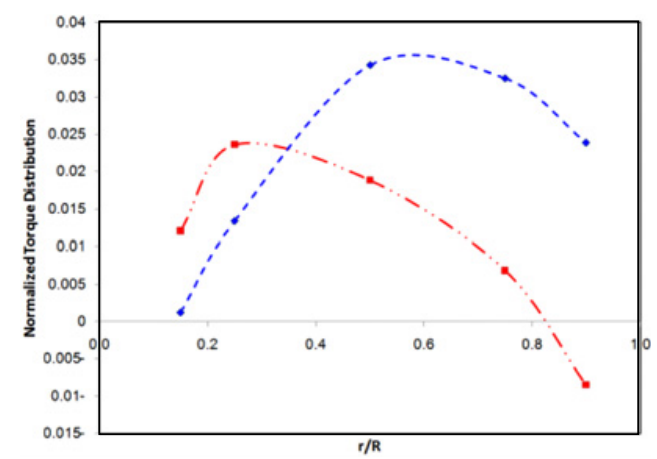

Figure 12. Comparison of the normalized torque for two CFD simulations.

The errors associated by the BEM can be attributed to the pitch angles which are different due to the complex nature of the three dimensional flows. The three dimensional flow alters the axial and rotational induction factors (a and a' in Fig. 1) and alter the relative flow angle and magnitude. The aerodynamic forces are proportional to the square of the relative flow angle and depend on the angle of attack. Errors in estimation of the aerodynamic forces result in the errors in power and thrust and complete CFD modeling is necessary to determine the turbine performance. Nevertheless, the BEM gives a good starting point for the CFD calculations.

\section{Conclusions}

The performance of a wind turbine was investigated using BEM and CFD. The BEM model was first used to design the wind turbine (chord distribution, twist, operating RPM, thickness) and compared to CFD solutions at select operating points. Comparing the power curve generated from the BEM to the CFD concludes that the BEM can be used in the variable speed operating zone. This is attributed to the fact that the most of the power is produced by the outer sections of the wind turbine which are essentially in two-dimensional flow. In the variable pitch operating zone, the results show significant deviation between the BEM and CFD. In this mode of operation the power is mostly produced from the inner sections which suffer from three-dimensional flow effects and cannot be modeled by the BEM. The deviation of the pitch angle between the BEM and CFD to maintain constant power after the rated wind speed can reach up to $10^{\mathrm{deg}}$. This is a significant angle and must be taken into consideration when designing pitch control systems for wind turbine blades. An experiment is underway to further validate the results of the CFD model.

This research was funded by the Science and Technology Development Fund (STDF) project ID 1705. The authors would like to thank Professor Galal Bahgat, Professor of Aerodynamics, Faculty of Engineering, Department of Aerospace for his support and insight.

\section{References}

[1] A. A. Afjeh and K. J. T. G., "A vortex lifting line method for the analysis of horizontal axis wind turbines," Transactions of the ASME, Journal of Solar Energy Engineering, 108, pp. 303-309, (1986) 
[2] F. J. Simoes and J. M. R. Graham, "A free vortex model of the wake of a horizontal axis wind turbine," Proceedings of 12th British Wind Energy Association Conference, pp. 161-165, (1990)

[3] G. van Bussel, The Aerodynamics of Horizontal Axis Wind Turbine Rotors Explored with Asymptotic Expansion Methods, $\mathrm{PhD}$ thesis, (1995)

[4] J. E Bardina, P. G. Huang and T. J. Coakly, "Turbulence Modeling Validation, Testing and Development," Tech report, NASA Technical Memorandum, (1997)

[5] L. Bermudez, A. Velazquez, and A. Matesanz, "Numerical Simulation of Unsteady Aerodynamics effects in Horizontal-axis Wind Turbines," Journal of Solar Energy 68, 9-21 (2000)

[6] P. R. Ebert, D. H. Wood, "The Near Wake of a Model Horizontal-axis Wind Turbine at Runaway," Journal of Renewable Energy 25 41-54 (2002)

[7] Duque, P. Earl, N. Burklund, D. Michael, and Johnson, Wayne, "Navier Stokes and Comprehensive Analysis Performance Predictions of the NREL Phase VI Experiment," Journal of Solar Energy Engineering 125, 457-467 (2002)

[8] P. Fuglsang, and C. Bak, "Development of the Risø Wind Turbine Airfoils," Wind Energy 7, P. 145-162 (2004)

[9] M. O. L. Hansen, and J. Johansen, "Tip Studies Using CFD and Comparison with Tip Loss Models," Wind Energy, P. 343-356 (2004)

[10] J. Johansen, H. A. Madsen, N. N. Sorensen, and C. Bak, "Numerical Investigation of a Wind Turbine Rotor with an Aerodynamically Redesigned Hub-Region," European Wind Energy Conference and Exhibition, Athens, Greece (2006)

[11] J. Johansen, and N. N. Sorensen, "Aerodynamic Investigation of Winglets on Wind Turbine Blades Using CFD," Ris $\varnothing-\mathrm{R} 1542$ (2006)

[12] A. Le Pape, and V. Gleize, "Improved Navier-Stokes Computations of a Stall Regulated Wind Turbine Using Low Mach Number Preconditioning," 44 ${ }^{\text {th }}$ AIAA Aerospace Sciences Meeting and Exhibit, Reno, NV, AIAA 1502 (2006)

[13] F. Zahla, J. Johansen, N. Sorenson, and J. Graham, "Wind Turbine Rotor-Tower Interaction Using an Incompressible overset Grid Method," AIAA 45 ${ }^{\text {th }}$ Aerospace Sciences Meeting and Exhibit, Reno, NV, AIAA 0425 (2007)

[14] Dnyanesh A. Digraskar, "Simulations of Flow Over Wind Turbines," Master of Science in University of Massachusetts Amherst (2010)

[15] I. Elqatary , B. Elhadidi, "Comparison of CFD with Two-Dimensional Strip Theory for Wind Turbine Simulation,” Open Source CFD International Conference, London (2012)

[16] www.salome-platform.org/

[17] http://wind.nrel.gov/designcodes/ 